\title{
Freiwillige Vasektomie in der Familienplanung - Gedanken zur Nutzen-Risiko-Analyse
}

\author{
W. Weidner, ${ }^{1}$, L. Weißbach ${ }^{2}$ \\ ${ }^{1}$ Klinik und Poliklinik für Urologie, Georg-August-Universität Göttingen (Direktor: Prof. Dr. R.-H. Ringert) \\ 2 Urologische Abteilung, Krankenhaus Am Urban, Berlin (Chefarzt: Prof. Dr. L. Weißbach)
}

\section{Zusammenfassung}

Die freiwillige Sterilitätsvasektomie ist ein Standardverfahren der Familienplanung. In die Entscheidungsfindung und Nutzen-Risiko-Abwägung sollte grundsätzlich die Partnerin miteinbezogen werden. Das Aufklärungsgespräch umfaßt die Möglichkeiten moderner Antikonzeption, operatives Vorgehen, peri- und postoperative Komplikationen, fragliche Nebenwirkungen (Gefäß- und Karzinomrisiko), postoperatives Verhalten (Konzeptionsschutz, Rekanalisierung) und Refertilisierungschance.

\section{Voluntary Vasectomy and Contra- ception: Analysis of Benefit and Risk}

Voluntary vasectomy is a standardized procedure of contraception. Decision-making and analysis of benefit and risk require intensive consultation involving the female partner. The information provided to the couple should include all modalities of modern contraception, the operative procedure, peri- and postoperative complications, questionable side effects (vascular and cancer risk), postoperative care (anticonception, recanalisation) and the chances of refertilization.

Key words

Vasectomy, Benefit-risk-analysis, Complications

\section{Einleitung}

Männer sehen sich immer häufiger einem wachsenden Druck ausgesetzt, einen größeren Beitrag zur Kontrazeption zu leisten. Dabei genießen sie nicht nur deren Vorteile, sondern setzen sich auch ihren Risiken aus. Insbesondere Entwicklungspolitiker erwarten einen männlichen Beitrag zur Eindämmung der Bevölkerungsexplosion: Die Weltbevölkerungskonferenz der Vereinten Nationen 1984 in Mexiko bezeichnet die männliche Kontrazeption durch Vasektomie als notwendig (Übersicht bei 19).

\section{Häufigkeit}

Es gibt nur spärliche Daten zur Vasektomie für den deutschsprachigen Raum. Während in den benachbarten Niederlanden und Großbritannien die Vasektomierate ca. $10 \%$ beträgt, soll in der Bundesrepublik Deutschland diese männliche Kontrazeptionsmaßnahme nur bei $3 \%$ liegen (zitiert bei 19). Eine bundesweite Umfrage zur Refertilisierung in der Bundesrepublik Deutschland hat gezeigt, daß durch Urologen ca. 25000 Sterilisationsvasektomien pro Jahr durchgeführt werden (7).

\section{Ziel der freiwilligen \\ Sterilitätsvasektomie}

Ziel des Eingriffs ist eine vollständige und sichere Blockierung der Spermatozoenpassage (33).

Dabei sollte die heute mögliche optimierte Reversionsmöglichkeit der Sterilitätsvasektomie (3) unseres Erachtens keine entscheidende Rolle bei der Zielsetzung des Eingriffs spielen. Die Gründe hierfür sind kürzlich von Wagenknecht (33) herausgearbeitet worden und werden von den Autoren dieses Beitrages vollinhaltlich geteilt: 1. Die Vasektomie ist ein operatives Verfahren zur dauerhaften Fertilitätskontrolle. 2. Die angestrebte Endgültigkeit erleichtert dem beratenen Paar den Entscheidungs- und Klärungsproze $\beta$ seiner Kontrazeptionsdiskussion. 3. Auch bei Durchgängigkeitsraten von 80-90\% nach Vasovasostomie werden nur $50-70 \%$ dieser Männer fertil $(3,33)$. Gründe für diese Diskrepanz liegen in zum Teil nur teilweise bekannten Mechanismen wie Druckveränderungen im Hoden, Auftreten von Spermatozoenantikörpern und eventuellen Hodengewebsveränderungen begründet. Sicher erscheint bisher nur, daß ein zunehmendes Intervall zwischen Vasektomie und Vasovasostomie die Refertilisierungsergebnisse bezogen auf die Schwangerschaft der Partnerin verschlechtert (3). 


\section{Operatives Vorgehen}

Es gibt kein standardisiertes Vorgehen zur Sterilitätsvasektomie (33).

Unseres Erachtens ist die Fulguration des Duktuslumens effektiv; sie wird nach Resektion von 1$2 \mathrm{~cm}$ Vas durchgeführt. Eine zusätzliche Interposition von Gewebe bzw. Decken der Samenleiterenden mit Faszienanteilen wird unterschiedlich beurteilt.

Das resezierte Ductusgewebe wird histologisch aufgearbeitet, um einen Identifikationsirrtum auszuschließen (17).

Grundsätzlich kann keine Standardtechnik der Vasektomie eine $100 \%$ ige Sterilität sichern (17). Langstreckige Resektionen (z.B. von mehr als $7 \mathrm{~cm}$ ) sind Verfahren, die uns nicht sinnvoll erscheinen (10), da auch sie keine $100 \%$ ige Sterilität garantieren.

\section{Peri- und postoperative Komplikationen}

Peri- und postoperativ sind die Risiken des Eingriffes gering. Todesfälle sind bei über 6 Mill. Eingriffen in den USA bisher nicht angegeben (19). Hämatome, Skrotalschwellungen und Infektionen im Operationsgebiet finden sich bei $1-6 \%$ der operierten Fälle $(17,19,33)$. Nach der auch von uns bevorzugten Fulguration des Lumens werden kurzzeitige Nebenwirkungen wie skrotale Schwellung, Hämatom und Infektion nur bei maximal $3 \%$ beobachtet (33). Eine floride Epididymo-Orchitis tritt bei weniger als $1 \%$ der Patienten auf $(14,19)$. In Ausnahmefällen kann noch Jahre nach Vasektomie eine Epididymitis auftreten, die auf einer massiven tubulären Dilatation des Nebenhodens beruht (25). Auch bei anhaltenden „Hodenschmerzen“ nach Vasektomie können zystische Nebenhoden-Veränderungen sonographisch nachgewiesen werden (16).

Es ist bekannt, daß nach Vasektomie Knoten im Bereich des Vasektomiebettes entstehen können. Diese sind als "Vasitis nodosa“ oder Sperm-Granulome bezeichnet worden (6). Die Pathogenese des Sperm-Granuloms ist nicht eindeutig, sie wird entweder als entzündliche Reaktion auf die Extravasation von Spermatozoen $(6,33)$ oder durch proliferierende kleine Kanälchen aus dem Lumen erklärt $(6,27)$. Bis heute ist ihre Entstehung nicht eindeutig abgeklärt und nicht voraussehbar (6). Andrologisch interessant ist die Tatsache, daß die Ausbildung von Sperm-Granulomen im Bereich des hodennahen Endes der Samenleiter oder des Nebenhodens positive Auswirkungen auf die Refertilisierungschance vasektomierter Männer haben soll (27).

\section{Operativer Fehlschlag}

Das Fehlschlagen einer Vasektomie als antikonzeptive Maßnahme ist selten $(6,10,17,24)$. Dabei wird ein Mißerfolg häufig durch eine ungewollte Schwangerschaft, selten durch eine Persistenz von Spermatozoen festgestellt (10). Üblicherweise dauert es in Abhängigkeit von der Ejakulationsfrequenz ein bis zwei Monate, bis keine Spermatozoen im Ejakulat mehr nachweisbar sind.
Dabei ist für uns der Nachweis einer Azoospermie, d.h. das Fehlen von Spermatozoen und Zellen der Spermatogenese nach Zentrifugation des Ejakulats 6 und 12 Wochen nach Vasektomie, entscheidend.

Mißerfolge der Vasektomie beruhen vermeidbar auf einem intraoperativen Identifikationsirrtum, extrem selten auf einer Mehrfachanlage des Vas deferens (17).

Häufigste Ursache, technikabhängig in bis zu $0,8 \%$, ist die spontane Rekanalisation. Für diese ist die Entstehung der o.g. Spermgranulome entscheidend (10, 17). Spätrekanalisationen nach Jahren sind extrem selten, jedoch nicht vermeidbar (Übersicht bei 24).

Daraus resultiert, daß durch die Vasektomie eine dauerhafte Sterilität nicht zu $100 \%$ erzielt werden kann.

\section{Langzeitrisiken}

\section{Arteriosklerose}

Vorhandene Skepsis gegenüber der Vasektomie ist vordergründig durch einen Bericht über eine erhöhte Inzidenz von diätetisch induzierter Arteriosklerose bei vasektomierten Affen geweckt worden (1). Die National Institutes of Health und die WHO haben große epidemiologische Studien in den USA bzw. in China veranlaßt, um nach Assoziationen zwischen Vasektomie und kardiovaskulären Erkrankungen (Gefäßrisikofaktor) $\mathrm{zu}$ fahnden (20). Zahlreiche weitere Studien (zitiert in 19) haben diese Untersuchungen erweitert, so daß die Vasektomie heute neben dem Rauchen der epidemiologisch am besten untersuchte Gefäßrisikofaktor ist. Alle Studien kommen übereinstimmend zu dem Schluß, daß vasektomierte Männer nicht nur im Hinblick auf Arteriosklerose und koronare Herzerkrankung, sondern auch auf Erkrankungen anderer Organsysteme, wenn nicht sogar eine niedrigere, so doch zumindest keine höhere Inzidenz aufweisen als nicht-vasektomierte Männer.

\section{Endokrine Veränderungen}

Vorbehalte, nach denen die Vasektomie im Verlauf des weiteren Lebens vermehrt zu Potenzstörungen führen könnte („androgene Vergreisung“), gehen auf experimentelle Studien an vasektomierten Ratten zurück (13). Hier fiel der Testosteronspiegel ab; Prostata- und Hodengewicht waren rückläufig. Es gibt auch Patienten, die nach dem Eingriff einen Rückgang der Potenz beklagen. Sorgfältige endokrinologische Untersuchungen haben jedoch keinen Einfluß auf den Testosteron- und Prolaktinspiegel sowie auf die Gonadotropine des Mannes erbracht (14). Etwaige hormonelle Veränderungen wären auch nicht zu erklären, da die Vasektomie die Produktion der Androgene langfristig nicht beeinflußt (32).

\section{Prostataerkrankungen}

Das Risiko, an einer benignen Prostatahyperplasie bzw. an einem Prostatakrebs zu erkranken, wird durch den Eingriff nicht erhöht (26). Neuere epidemiologische Daten, die ein geringfügig erhöhtes alterskor- 
rigiertes Risiko bei Vasektomierten für das Prostatakarzinom zeigen, beruhen wahrscheinlich auf der besonders sorgfältigen Nachsorge der vasektomierten Männer in dieser Studie (22).

\section{Hodenkarzinom}

Verschiedene Arbeiten stellen einen $\mathrm{Zu}-$ sammenhang zwischen Vasektomie und Hodenkrebs her, obwohl wegen der geringen Inzidenz (2-8 auf 100000) epidemiologische Aussagen über den Hodentumor von vornherein schwierig sind und als Risikofaktoren bisher nur die angeborene Hodenfehllage und die vorausgegangene Erkrankung an einem Hodentumor bekannt sind. In einer retrospektiven Erhebung an einem schottischen Krankenhaus wurden 3079 vasektomierte Männer kontrolliert (4): Nach einer Zeit von 0,25-4 Jahren erkrankten 8 an einem Hodentumor, während das kalkulierte Risiko mit 1,9 Erkrankungen deutlich niedriger errechnet worden war. Eine irische Arbeitsgruppe entdeckte bei den zwischen 1980 und 1985 vasektomierten Männern 3 mit einem Hodentumor; sie waren innerhalb von 8 Wochen nach der Operation diagnostiziert worden (31). Schließlich führten in Washington Strader et al. zur Ermittlung der Tumorinzidenz 333 Telefoninterviews mit Männern durch, bei denen zwischen 1977 und 1983 ein Hodentumor diagnostiziert worden war (28). Gleichzeitig wurden in einer Kontrollgruppe 729 Männer auf gleiche Weise befragt. Die Tumorinzidenz lag auch hier bei den Vasektomierten höher. Überraschend fanden die Autoren einen Zusammenhang mit der Religionszugehörigkeit: In der Kontrollgruppe waren 19\% der Vasektomierten Protestanten, aber nur 6,2\% Katholiken. Das deutet lediglich auf eine fehlende Offenbarungsbereitschaft der katholischen Männer über die vorangegangene Vasektomie hin, denn die höhere Tumorinzidenz war nur bei den Katholiken gefunden worden.

Diese Untersuchungen ermöglichen bestenfalls folgende Aussagen: bei den zur Vasektomie anstehenden Männern kann offenbar ein Carcinoma in situ vorbestehen, das sich nach dem Eingriff manifestiert. Deshalb sollten diese Männer über die Möglichkeit der palpatorischen Selbstkontrolle aufgeklärt werden. Ob ein Carcinoma in situ durch immunologische Prozesse oder durch postoperative intratubuläre Druckerhöhungen aktiviert werden kann, bleibt unklar. Studien über die Hodenpathologie vasektomierter Tierspezies zeigen starke Unterschiede (Übersicht bei 15), und es ist zweifelhaft, ob diese Ergebnisse auf den Menschen übertragbar sind. Hodenbiopsien vasektomierter Männer (15) zeigen $u$. a. Erweiterungen der Tubuli seminiferi, die einen erhöhten intraluminalen Druck suggerieren. Eine kausale Verknüpfung dieses Befundes mit den diskutierten Folgen ist spekulativ (12).

Zusammenfassend bietet sich eine Erklärung für eine testikuläre Tumorentwicklung nach Vasektomie nicht an. Die Beobachtungen können Zufallsbefunde sein und beweisen weder die Entstehung eines Hodentumors durch Vasektomie, noch lassen sie sich durch die kleinen Fallzahlen statistisch absichern. Deshalb sind prospektive Untersuchungen zu fordern, um das Risiko der Entwicklung von Hodentumoren bzw. deren Aktivierung durch die Vasektomie besser abschätzen zu können.

\section{Spermatozoenantikörper und Fertilitätschance}

Sicher ist, daß nach der Vasektomie Spermatozoenantikörper auftreten (9). Sie beeinflussen den Erfolg einer eventuellen Refertilisierungsoperation (19). Bis auf gehäufte Epididymo-Orchitiden (14) bestehen keine anderweitigen Wechselwirkungen zum Gesamtorganismus (z. B. Induktion von Autoaggressionskrankheiten).

Für die Refertilisierung werden die gegen Spermatozoen gebildeten Antikörper als Fertilitätshindernis akzeptiert (Übersicht bei 19). Die Titerhöhe ist jedoch umstritten, wobei die Erhöhung der Spermatozoenantikörper auch mit entzündlichen Veränderungen im Bereich des peripheren Stumpfes des Vas deferens korreliert worden ist (2).

Eine wichtige Rolle für die Refertilisierungschance spielen auch intraluminale Druckerhöhungen. Bei einem exokrinen Organ sollte man nach Verschluß des Ausführungsganges eine Zerstörung befürchten. Die Organintegrität des Hodens beruht vor allem auf der hohen Pufferkapazität zur Rückresorption von Flüssigkeit im Nebenhoden (Übersicht bei 19). Dennoch führt der Eingriff wahrscheinlich neben morphologischen Veränderungen des Nebenhodens auch zur Verschlechterung der Spermatogenese (11). Beobachtungen, daß nach Vasektomie auftretende Sperm-Granulome die Refertilisierungschance günstig beeinflussen (27), werden so gedeutet, daß diese quasi als „Sicherheitsventil“ zu einer intraluminalen Druckentlastung führen. Von diesen Beobachtungen ausgehend, sind einige Urologen dazu übergegangen, die "Open-End-Vasektomie“ durchzuführen. Dabei wird der distale Stumpf verschlossen, während der proximale hodennahe Stumpf offen bleibt (8). Langfristig erscheint die Chance zur Wiedererlangung der Fertilität so vasektomierter Patienten besonders erfolgversprechend (Übersicht bei 19).

\section{Psycho-sexuelle Auswirkungen}

Eine GALLUP-Umfrage stellte 1979 fest, daß nur 25 Prozent der amerikanischen Männer ernsthaft eine Vasektomie zur Familienplanung in Betracht ziehen würden (18). Ursache hierfür sei in vielen Fällen die Meinung, eine Vasektomie habe einen „entmannenden“ (androgene Vergreisung) Effekt, der sachlich nicht begründet werden kann (Übersicht bei 30).

Auch der immer wieder angeführte Verlust an Ejakulatmenge nach Vasektomie (18) ist nicht nachvollziehbar, da $80-95 \%$ des Samenergusses aus Prostata, Bläschen- und Cowperschen Drüsen sezerniert werden. Neuere Untersuchungen haben ergeben, daß sich tatsächlich die Ejakulatvolumina vor und nach Vasektomie nicht unterscheiden (Weiske, persönliche Mitteilung).

Es wurde weiter diskutiert, daß einige Frauen glauben könnten, eine Vasektomie würde dem Partner Chancen bieten, ohne Gefahr einer Schwangerschaft außerehelich aktiv zu werden. Untersuchungen an Ehepaargruppen, die Antikonzeption mit Ovulationshemmern bzw. Vasektomie betrieben, haben jedoch bereits in 
den sechziger Jahren gezeigt, daß derartige Auswirkungen der Vasektomie nicht bestehen (21).

Im allgemeinen findet sich in der Literatur die Auffassung einer Zunahme der sexuellen Aktivität und Libido nach „endgültiger“ Regelung der Familienplanung durch Vasektomie (Übersicht bei 23), wobei eine stärkere Zuwendung der Partner im Vordergrund steht.

\section{Aufklärung}

Aus diesen Ausführungen folgert, daß vor jeder freiwilligen Sterilitätsvasektomie zur Familienplanung eine umfassende Aufklärung des Paares über moderne Antikonzeption notwendig ist.

In die Entscheidungsfindung und NutzenRisiko-Abwägung sollte unseres Erachtens grundsätzlich die Partnerin miteinbezogen werden. Der seltene Wunsch nach Vasektomie ohne Information der Partnerin $(18,23)$ wird von uns nicht erfüllt. Grundlage dieser Haltung ist auch die Empfehlung des Bundesgerichtshofes, sich nicht mit der zivil- wie strafrechtlich maßgeblichen Einwilligung des sterilisationswilligen Mannes zu begnügen (29).

Das gemeinsame Gespräch sollte nachfolgende Punkte umfassen und schriftlich niedergelegt sein:

1. Antikonzeption heute,

2. operatives Vorgehen,

3. Komplikationen (peri- und postoperativ),

4. Nebenwirkungen (Kurzzeit- und Langzeit-),

5. postoperatives Verhalten (Konzeptionsschutz,

Spermiogrammkontrollen, Rekanalisierung),

6. Refertilisierungschance.

Bei einem derartigen Vorgehen wird das seit 20 Jahren etablierte Verfahren der Vasektomie im Rahmen der Familienplanung seinen wichtigen Stellenwert behalten.

\section{Literatur}

1 Alexander, N. J., T. B. Clarkson: Vasectomy increases the severity of diet - induced atherosclerosis in Macaca fascicularis. Science 201 (1978) 538-541

2 Bandhauer, K., E. Senn: Die Vaso-Vasostomie-Faktoren, welche die Ergebnisse beeinflussen. Akt. Urol. 18 (1987) 7-10

3 Belker, A. M., A. J. Thomas, E. F. Fuchs, J. W. Konnak, J. D. Sharlip: Results of 1469 microsurgical vasectomy reversals by the vasovasostomy study group. J. Urol. 145 (1991) 505-511

4 Cale, A. R. J., M. Farouk, R. J. Prescott, I. W. J. Wallace: Does vasectomy accelerate testicular tumour? Importance of testicular examinations before and after vasectomy. Brit. Med. J. 300 (1990) 370

${ }^{5}$ Cos, L. R., J. R. Valvo, R. R. Davis, A. T. K. Cockett: Vasovasostomy, current state of the art. Urology 22 (1983) $567-575$

${ }^{6}$ Cruishank, B., L. Eidus, M. Barkin: Regeneration of vas deferens after vasectomy. Urology 30 (1987) 137-142

7 Engelmann, U. H., P. Schramek, G. Tomamichel, F. Deindl, Th. Senge: Vasectomy reversal in central europe; results of a questionnaire of urologists in Austria, Germany and Switzerland. J. Urol. 143 (1990) 64-66

8 Errey, B. B., I. S. Edwards: Openended vasectomy. An assessment. Fertil. Steril. 45 (1986) 843-845
${ }^{9}$ Fuchs, E. F, N. J. Alexander: Immunologic considerations before and after vasovasostomy. Fertil. Steril. 40 (1983) 497 499

10 Hallan, R. I., A. R. L. May: Vasectomy: how much is enough? Brit. J. Urol. 62 (1988) 377-379

11 Jarow, J. P., R. E. Budin, B. Dym, R. Zirkin, S. Noren, F. F. Marshall: Quantitative pathologic changes in the human testis after vasectomy. New Engl. J. Med. 313 (1985) 1252-1256

12 Jorgensen, N., A. Giwercman, S. W. Hansen, N. E. Skakkeboek: Biological mechanisms for a relation between vasectomy and testicular cancer. Onkologie 14 (Suppl. 4) (1991) 5-6

13 Kinson, G. A., R. A. Layberry: Long-term endocrine responses to vasectomy in the adult rat. Contraception 11 (1975) $143-$ 150

14 Massey, F. J. and STUDY GROUP: Vasectomy and health J. Amer. med. Ass. 252 (1984) 1023-1029

15 McDonald, St. W.: Vasectomy and the human testis. Br. Med. J. 301 (1990) 618-619

16 McMahon, A. J., J. Buckley, A. Taylor, S. N. Lloyd, R. F. Deane, D. Kirk: Chronic testicular pain following vasectomy. Brit. J. Urol. 69 (1992) 188-191

17 Melchior, H., J. Sökeland, W. Weissauer: Freiwillige Sterilisations-Vasektomie. Dt. Ärzteblatt 88 (1991) B 1837-1838

18 Mumford, St. D.: Vasektomie und Vasektomie-Beratung. In Swanson, Forrest (Hrsg.): Die Sexualität des Mannes. Dtsch. Ärzteverlag Köln (1987) 211

${ }^{19}$ Nieschlag, E.: Vasektomie - pro und contra. Dtsch. med. Wschr. 112 (1987) 1107-1109

20 Perrin, E. B., J. S. Woods, T. Namekuta, J. Yagi, R. A. Bruce, $V$. Hofer: Long-term effect of vasectomy on coronary heart disease. Amer. J. Publ. Hlth. 74 (1984) 128-132

21 Rogers, D. A., F. J. Ziegler: Changes in sexual behavior consequent to use of noncoital procedures of contraception. Psychosomatic Medicine 30 (1968) 495-498

22 Rosenberg, L., J. R. Palmer, A. G. Zauber, M. E. Warshauer, $P$. D. Stolley, S. Shapiro: Vasectomy and the risk of prostate cancer. Amer. J. Epidemiol. 132 (1990) 1051-1055

23 Schirren, C.: Vasektomie - Pro und Kontra. TW Urologie Nephrologie 2 (1990) $247-252$

24 Schwab, M., H.-H. Sparwasser: Spätrekanalisation nach Vasoresektion. Urologe B 31 (1991) 12-15

25 Selikowitz, S. M., A. R. Schned: Late post-vasectomy-syndrome. J. Urol. 134 (1985) 494-497

26 Sidney, St.: Vasectomy and the risk of prostatic cancer and benign prostatic hypertrophy. J. Urol. 138 (1987) 795-797

27 Silber, S. J.: Vasectomy and vasectomy reversal. Fertil. Steril. 29 (1978) 125-140

28 Strader, C. H., N. S. Weiss, J. R. Daling: Vasectomy and the incidence of testicular cancer. Amer. J. Epidem. 128 (1988) 56 $-63$

29 Thon, W. F., C. G. Stief, U. Jonas: Vasektomie: kleiner Eingriff - große Folgen. Urologe A 31 (1992) 55-57

30 Thonneau, $P$., $B$. D'Isle: Consequences à long terme de la vasectomie: revue de la litterature. Andrologie (Lille) 1 (1991) 26 $-28$

31 Thornhill, J. A., M. Butler, J. M. Fitzpatrick: Could vasectomy accelerate testicular cancer? Brit. J. Urol. 59 (1987) 367

32 de la Torre, B., M. Hedman, F. Jensen, P. H. Pedersen, E. Diczfalusy: Lack of effect of vasectomy on periphereal gonadotrophin and steroid levels. Int. J. Androl. 6 (1983) 125-134

33 Wagenknecht, L. V.: Ambulante Sterilisation beim Mann: Technik und Komplikationen der Vasoresektion. Fertilität 7 (1991) $85-89$

\section{Prof.Dr. W. Weidner}

Urologische Klinik

Georg-August-Universität Göttingen

Robert-Koch-Str. 40

3400 Göttingen 